\title{
Energy and Delay Efficient Duty-Cycle MAC Protocol for Multi-hop Wireless Sensor Networks
}

\author{
Seong Cheol Kim ${ }^{1}$, Jun Heon Jeon ${ }^{1}$, Joong Jae Kim ${ }^{1}$ \\ ${ }^{1}$ Sangmyung University, Seoul, South Korea \\ sckim@smu.ac.kr,cloud0305@naver.com,Joongjae.kim@gmail.com
}

\begin{abstract}
Low energy consumption has always been the most important main issue in wireless sensor networks. But many applications requiring low latency and high throughput have been emerged recently. Many existing duty-cycle MAC protocols such as S-MAC [1] and T-MAC [2] provide good power efficiency but introduce significant end-to-end delay. In this paper, we proposed an energy and delay efficient duty-cycle MAC protocol which reduces data transmission delay from source node to destination node and node energy consumption in multi-hop Wireless Sensor Networks. The main goal is to design a MAC protocol for object tracking application, in which large amounts of data need to be transmitted when an event occurs. An operation cycle of the proposed MAC protocol is consists of four periods as in LO-MAC [10]. But in the proposed MAC protocol all the nodes in the data path repeat the Sleep periods only when an event occurs. And during Data period nodes immediately go into sleep mode with overhearing the PION packet. Performance evaluation shows that the proposed MAC protocol improves the transmission delay and the throughput compared to the RMAC.
\end{abstract}

Keywords: Energy consumption, Latency, Multi-hop, MAC protocol, WSNs

\section{Introduction}

Wireless sensor networks (WSNs) consist of large large number of small sensor nodes and are interconnected in an ad-hoc manner to transmit data from many sensor nodes to the destination node, in other words, sink node. But these small sensor nodes use batteries and replacing the batteries may be difficult. So it is very important to use sensor node energy efficiently to prolong the network life time. One of effective methods to save sensor node energy is to implement low power technique at Medium Access Control (MAC) [1-4]. Many MAC protocols are suggested for this purpose. Some protocols use duty-cycling mechanism to save energy. In duty cycling MAC protocols, sensor nodes repeat wakeup and sleep period. In sleep period nodes turn their power off and save energy. The main problem in these protocols is to increase the packet transmission delay [5-7]. Since nodes in a sleep state do not act in data transmission, so packet transmission delay from source node to destination increases. Therefore in real-time applications like surveillance or environments monitoring, the duty cycling mechanism is not adequate. Providing end-to-end delay guarantee for multihop wireless sensor networks is critical issues in these applications. In this paper we suggest an MAC protocol which may provide end-to-end data transmission requirement and reduce node energy consumption for multi-hop wireless sensor networks. In the proposed MAC protocol, nodes in a path from source node to destination follow different entire time periods depending on the data traffic patterns. This paper is an extension of our previously published paper [12]. The rest of this paper is organized as follows. Section 2 summarizes the related 
works on providing end-to-end delay in multi-hop wireless sensor networks. Section 3 introduces our MAC protocol and performance evaluation of our MAC protocol is carried out in Section 4. Finally, Section 5 concludes this paper.

\section{Related Works}

The MAC protocols of WSNs may be divided into three categories; synchronous MAC protocol, asynchronous MAC protocol, and hybrid MAC protocol. The proposed MAC protocol in this paper belongs to a synchronous MAC protocol. Examples of the related synchronous MAC protocols are RMAC [8], DW-MAC [9], and LO-MAC [10]. We describe here these MAC protocols briefly.

\subsection{RMAC Protocol [8]}

A Routing-enhanced duty cycle MAC (RMAC) protocol has Sync, Data, and Sleep periods and uses Pioneer Control Frame (PION) packet to determine multi-hop path. This PION packet includes the duration of the transmission, the current node, and the next node's address. The PION also has cross-layer information such as final destination node address and the number of hops. Data packet transmission between nodes in RMAC protocol is done during Sleep period and an ACK packet from receiver node is required. RMAC protocol uses cross-layer information for routing and works well in low data rate network environments. Figure 1 shows the operation of the RMAC protocol. As shown in the figure PION packet is transmitted during DATA period and data packet is sent to its destination during SLEEP period. All nodes except nodes in participating data transmission go to sleep mode to reduce energy consumption.

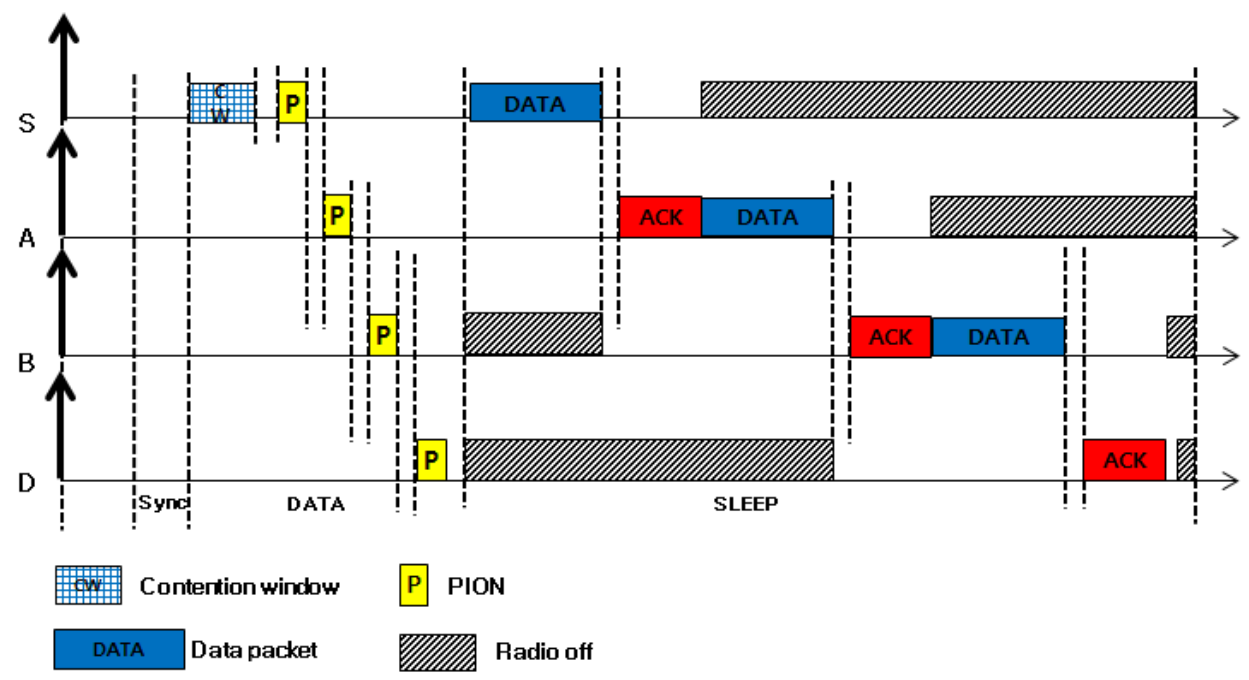

Figure 1. A Routing-enhanced Duty Cycle MAC (RMAC) Protocol

\subsection{DW-MAC Protocol [9]}

Demand Wakeup MAC (DW-MAC) protocol is a synchronous MAC protocol in a multi-hop WSNs. The legacy synchronous MAC protocols such as S-MAC [1] and TMAC [2] do not support multi-hop data transmission. Even though the data packets are 
transmitted during the SLEEP periods, the collisions in a RMAC may occur. In RMAC a source node always try to send its packet at the beginning of a SLEEP period. So packets from two sources may collide at the beginning of the next SLEEP period. This collision problem can be resolved by using scheduling mechanism in DW-MAC protocol. DW-MAC protocol reduces the risk of collisions during SLEEP period by a one-to-one mapping between a DATA and the SLEEP period. DW-MAC protocol also has fixed Sync, Data, and Sleep periods and uses scheduling frame $(\mathrm{SCH})$ to support efficient multi-hop data transmission.

\subsection{LO-MAC Protocol [10]}

The Low overhead MAC (LO-MAC) protocol works like the RMAC protocol except using Carrier sensing (CS) period. The CS period is added to Sync, Data, and Sleep periods. During CS period a node with pending data uses busy tone and only busy tone received nodes wakeup to take part in the data transmission. Data transmission can be done during Sleep period like in RMAC but acknowledgement for the received packet can be done using overhearing. This can reduce data transmission time and energy consumption.

\section{Design}

\subsection{Overview}

The proposed MAC in this paper is designed for applications such as object tracking and wild animal monitoring. In these applications an event occurring is not common. So a low data transmission is usually carried out. But when an event occurs, continuous object tracking observation is required. This generates lots of data transmission from the sensor nodes to sink node. So DW-MAC, RMAC, and LO-MAC are not adequate for this environment. Figure 2 shows the overview of a frame of the proposed MAC protocol. As shown in the figure a frame is composed of five periods; Sync, CS 1, DATA, SLEEP, and CS 2.

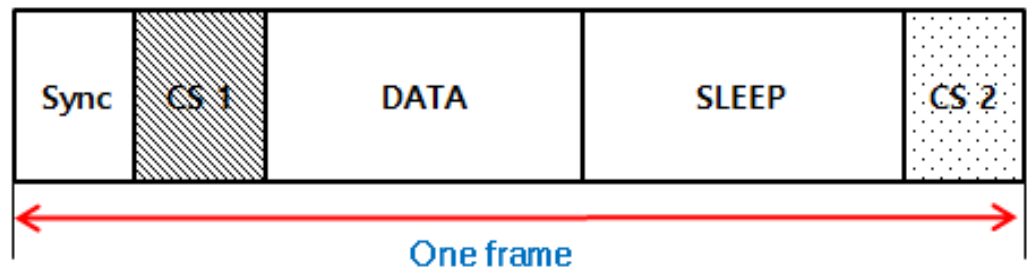

Figure 2. Overview

\subsection{Multi-hop Transmission}

When a source node has data packets to send, it will first send a busy tone in CS (Carrier Sensing) period. And the source node sends a PION packet to its neighbor node during Data period. The PION packet will be delivered to its final destination node. The PION packet contains the following information: Current node's address, next node's address, NAV (as in IEEE 802.11[11]), the number of hops, and the destination of the current flow [9]. Data packet transmission is done during Sleep period through the route, which was determined with PION packet. If the source node gets more data to send, then it sends a busy tone to its 
child node during CS2 period [12]. This busy tone will be transmitted to the final sink node and all the nodes along the path know another data transmission. So an additional data transmission can be done without repeating Sync, CS1, and Data periods. So total data transmission delay can be reduced.

\subsection{Energy-efficient Mechanism}

The main purpose of the proposed MAC protocol is to enhance the RMAC and LO-MAC protocol. So the proposed MAC protocol adapts similar characteristics of RMAC and LOMAC. In other words, the proposed MAC protocol uses the PION packet, and the same operation cycle, which is divided into Sync, Data, and Sleep periods like in RMAC. It also uses CS period and overhearing for acknowledgement from LO-MAC protocol. However, the proposed MAC protocol works differently when an event occurs and burst data should be transmitted. In this case only Sleep periods are repeated. The proposed MAC protocol can save more energy by using early sleep mode. This early sleep mode can be done during Data period. In RMAC and LO-MAC protocols, a PION packet is delivered to its final destination node during Data period. After sending a PION packet to its child node, a source node stays in wakeup mode until Sleep period starts without doing anything. And the intermediate nodes also stay in wakeup mode. In the proposed MAC protocol a sender node goes to sleep mode after sending PION packet and overhearing the transmission between intermediate nodes. All other intermediate nodes also go into sleep mode early, so energy consumption can be reduced. The operation of the proposed MAC protocol is shown in the figure 3.As shown in the figure, sleep mode insertion of the source and intermediate nodes in the Data period and the repeated Sleep period is the key idea of the proposed MAC protocol. All the intermediate nodes stay in radio-off until receiving packets and go immediately into sleep mode to save energy consumption like RMAC protocol.

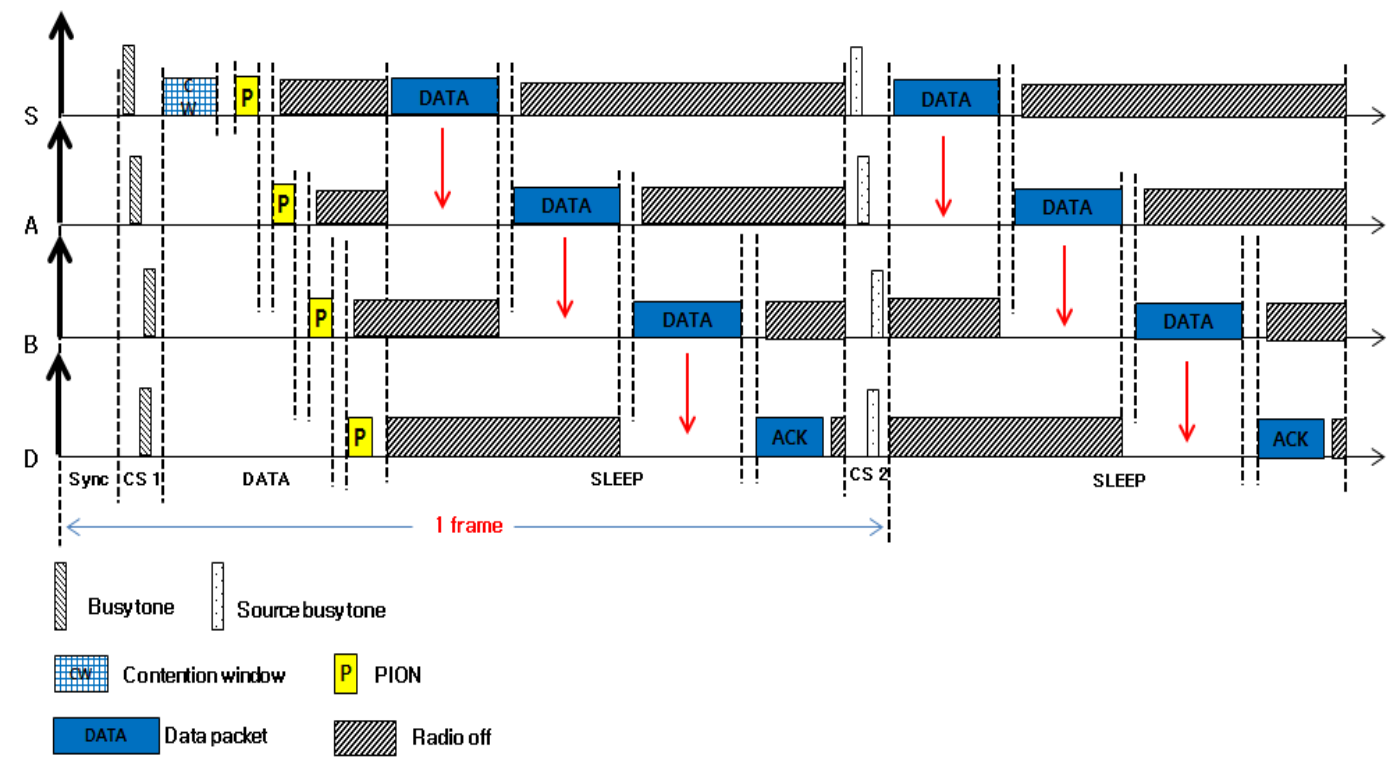

Figure 3. Mechanism 


\subsection{Algorithm}

Source node's algorithm of the proposed MAC protocol is as follows;

\section{1: Node wakeup}

2: SYNC exchange

3: if send data $=$ ! Null

broadcast $=$ busy tone signal

if received $==$ busy tone signal

broadcast $=$ busy tone signal

4: waiting $C W$

5: transfer $=$ PION

6: overhearing next node transfer PION

7: Sleep until Data period

8: wakeup

9: data packet transmission

10: overhearing next node transfer data packet

11: Sleep until Sleep period

12: wakeup

13: if send data $=$ ! Null

broadcast $=$ busy tone signal

goto 9

else goto 2

14: end

The proposed MAC protocol is a synchronous MAC protocol like S-MAC. In a synchronous MAC protocol all sensor nodes have the same schedule, so wakeup same time and exchange Sync signals each other. The proposed MAC protocol is a duty cycling contention-based MAC protocol. At the beginning, all nodes have the same operational cycle. This operational cycle is divided into 5 periods; Sync, CS1, Data, Sleep, and CS2. During CS1 period, all nodes with pending data packets broadcast their busy tone to all their neighbor nodes. The busy tones may collide but it does not matter. Busy tone collision indicates that there exists any node which has data packet to send. All nodes that receive a busy tone signal will stay in a wakeup state. A source node sends PION packet to its child node during Data period as shown in figure 2. After receiving the PION packet, the child node A resends the PION packet to node B. After overhearing the PION packet from node A to B, the source node immediately goes into sleep mode to save energy consumption. This is different from LO-MAC, in which the source node stays wakeup mode until Data period starts. When a PION packet arrives at the destination node, a path from source node to destination node is 
established. All data packets are transmitted during the Sleep period. In the beginning of a Sleep period, a source node sends data packet to node A as shown in the figure 2 . And after overhearing the data transmission from node A to node B, the source node goes into sleep mode. The overhearing indicates that the packet transmission was successful. The same data packet transmission continues to the destination node. After the data packet arrived at the destination node, in other words, the data transmission was successful, all the nodes in the data path wakeup at CS period. In the CS2 period, only a source node can send busy tone signal if the source node has data packet to send. This CS2 period is useful when an event like object appearing occurs. In this case, continuous object monitoring is required. And large bursts of data packets may be generated. So the source node needs to keep track the object and collects data, and sends data packets. Without repeating an entire periods, only the CS2 and Sleep period repeated using the same path from source node to destination node. This feature can support high throughput and low delay data transmission when an event occurs. But if an event does not occur and the source node does not have data packet to send, then the source node does not send busy tone.

\section{Performance Evaluation}

Table 1. Network Parameters

\begin{tabular}{ll}
\hline Parameters & Value \\
\hline Bandwidth & $20 \mathrm{kbps}$ \\
Rx power & $0.5 \mathrm{~W}$ \\
Tx range & $250 \mathrm{~m}$ \\
Tx power & $0.5 \mathrm{~W}$ \\
Idle power & $0.45 \mathrm{~W}$ \\
Sleep power & $0.05 \mathrm{~W}$ \\
Carrier sensing range & $550 \mathrm{~m}$ \\
Contention window & $64 \mathrm{~ms}$ \\
DIFS & $10 \mathrm{~ms}$ \\
\hline
\end{tabular}

Table 2. Transmission Parameters

\begin{tabular}{lll}
\hline Parameter & Frame Size(byte) & Tx Latency $(\mathrm{ms})$ \\
\hline RTS / CTS & 10 & 11.0 \\
Ack & 10 & 11.0 \\
PION & 14 & 14.2 \\
DATA & 50 & 43.0 \\
\hline
\end{tabular}


In this section performance of the proposed MAC protocol is compared to the related works based on analytical computation. We first considered a low data rate environment, in which only a data packet is transmitted from source node to the destination node. And then considered a high data rate environment, in which burst data packets are transmitted. Burst data packets may be generated when an event occurs in an application like animal monitoring application. We used the same parameters used in [9]. Table 1 and table 2 shows the used simulation parameters.

\subsection{Low Rate Traffics Environment}

We first considered a low traffic and load environments. In this environment we assume that a source node periodically generates a few data packet frames. Comparison of the average energy consumption of each sensor node is show in the Figure 4. As shown in the figure, we found that the proposed MAC protocol used less energy compared to the RMAC. The main difference comes from the fact that a node goes immediately into sleep mode after sending PION in the proposed MAC protocol.

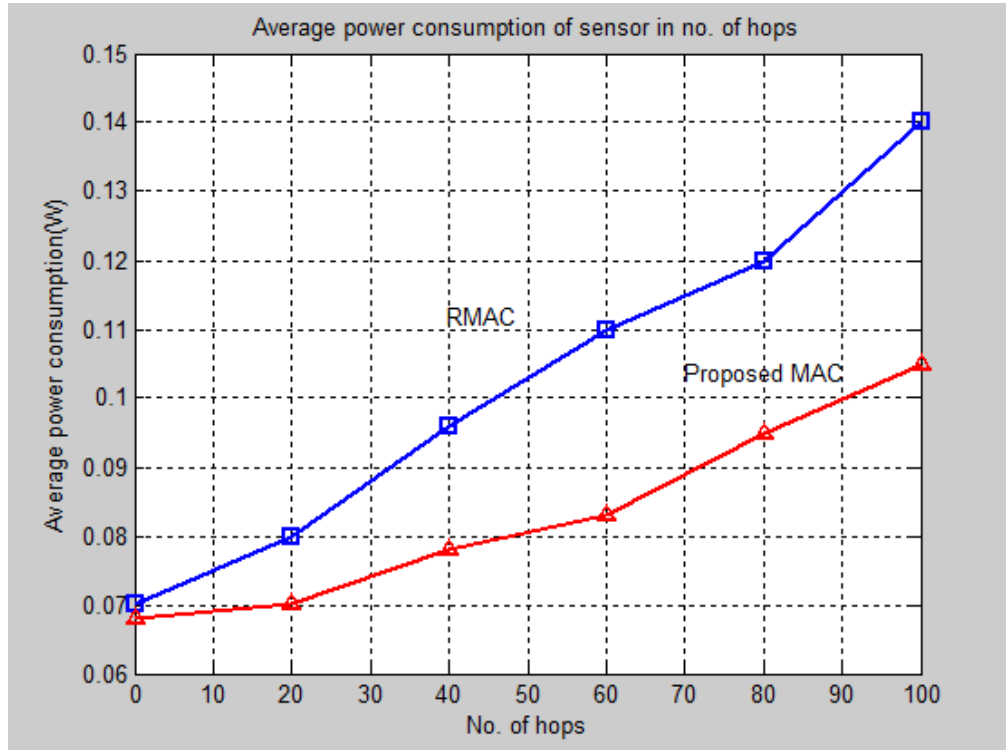

Figure 4. Average Power Consumption of Sensor with no. of Hops

\subsection{High Traffics Environment}

We considered here a high traffic load environments for Wireless Sensor Networks. In this environment we assumed that source nodes generated random number of data packets from 1 to 7. Comparison of the average energy consumption of each sensor node is shown in the Figure 5. As shown in the figure we found that our MAC protocol showed better energy saving than other protocols. Also comparison of the average delay of data packet transmission is shown in the Figure 6. As shown in the figure we found that our transmission method showed better low latency than other methods. 


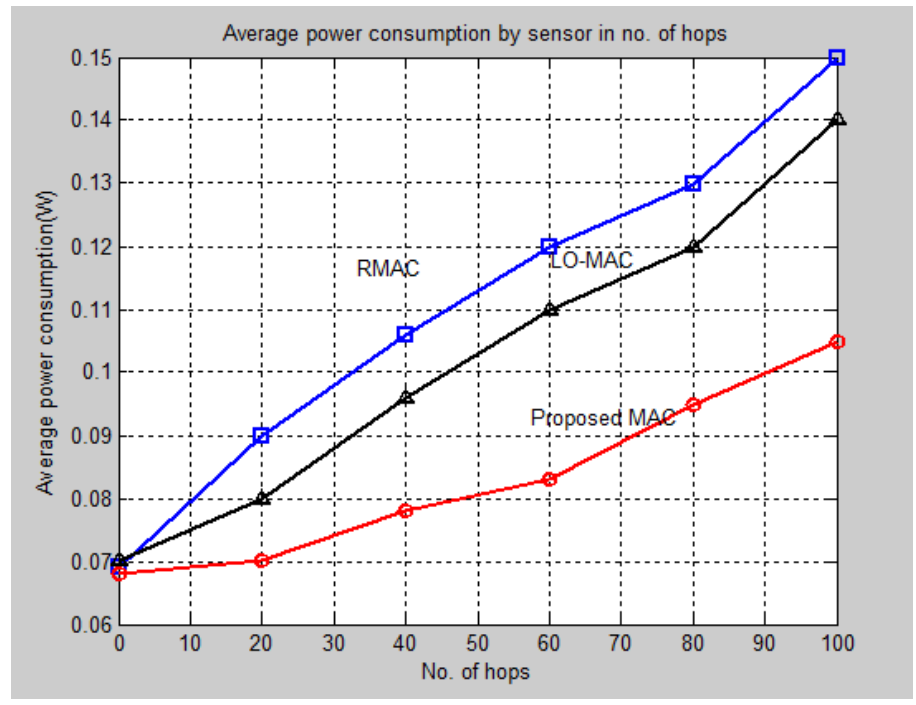

Figure 5. Average Power Consumption of Sensor with no. of Hops

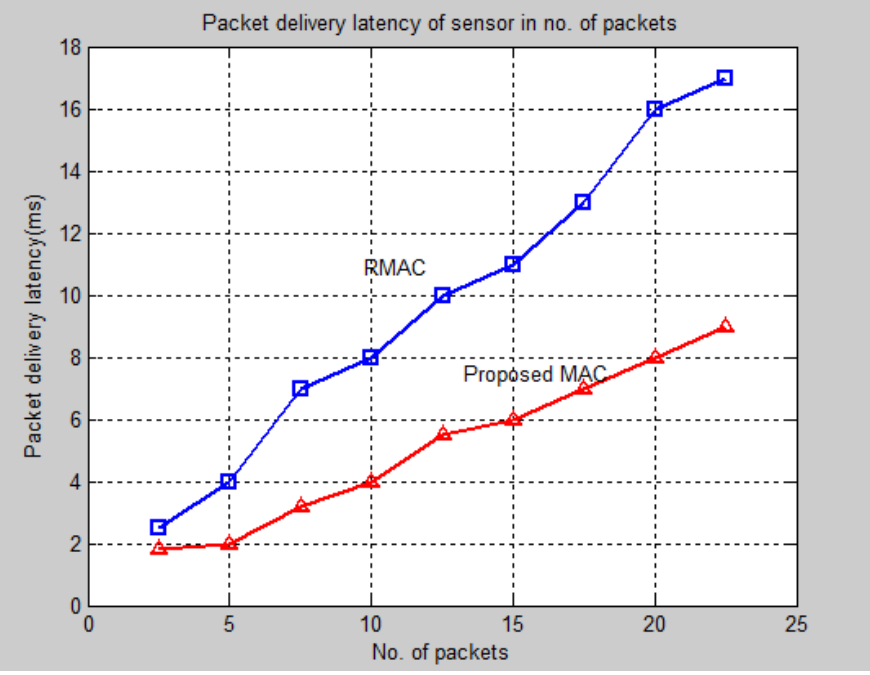

Figure 6. Packet Delivery Latency of Sensor with no. of Packets

\section{Conclusion}

In this paper, we presented an energy efficient low latency MAC protocol for multihop Wireless Sensor Networks. The proposed MAC protocol was designed for the applications such as object tracking and wild animal monitoring. In these applications, low data rate transmission is usually carried. But when an object appears, large amounts of data traffics are generated and source nodes have to send these data as soon as possible to the destination node. So multiple data packets are required to be sent from source nodes to a destination node, or a sink node in a limited time. For this purpose the proposed MAC protocol uses a duty cycling contention mechanism. When an event occurs only data transmission periods may be extended to send more data quickly, so transmission delay can be reduced and energy consumption may be reduced. We compared our MAC with RMAC and LO-MAC through extensive simulation. We found 
that our MAC outperforms these MAC protocols, with low latency, and low energy consumption as no. of hops increase.

\section{Acknowledgements}

This research was supported by a 2013 Research Grant from Sangmyung University.

\section{References}

[1] W. Ye, J. Heidemann and D. Estrin, Medium Access Control with Coordinated Adaptive Sleeping for Wireless Sensor Networks, IEEE/ACM Transactions on Networking, Vol.12, Issue 3, pp.493-506, June (2004).

[2] T. Dam and K. Langendoen, An Adaptive Energy-Efficient MAC Protocol for Wireless Sensor Networks, ACM Sensys'03, USA (2003).

[3] B. Manju and A. Lalit, Proficient D-HEED Protocol for Maximizing the Lifetime of WSN and Comparative Performance Investigations with Various Deployment Strategies, International Journal of Advanced Science and Technology (IJAST) Volume 45, pp 107-124, August (2012).

[4] S. C. Kim, J. H. Jeon and H. J. Park, QoS Aware Energy-Efficient (QAEE) MAC Protocol for Energy Harvesting Wireless Sensor Networks, Convergence and Hybrid Information Technology 2012, LNCS, vol. 7425, pp. 41-48, Springer, (2012).

[5] R. Naveed and H. Mureed, Ad hoc wireless Sensor Network Architecture for Disaster Survivor Detection, International Journal of Advanced Science and Technology (IJAST) Volume 34, pp 9-16, September (2011).

[6] M. Z. Hussain, M. P. Singh and R. K. Singh, Analysis of Lifetime of Wireless Sensor Network, International Journal of Advanced Science and Technology (IJAST) Volume 53, pp 117-126, April (2013).

[7] S. C. Kim, J. H. Jeon and H. J. Park, Energy Efficient Data transmission Mechanism in Wireless Sensor Networks, Lecture Notes in Electrical Engineering, vol. 235, pp 845-852, Springer, (2013).

[8] S. Du, A. Saha and D. Johnson, RMAC: a routing-enhanced duty-cycle MAC protocol for wireless sensor networks, Proceedings of the 26th Annual IEEE Conference on Computer Communications (INFOCOM 2007), pp.1478-1486, May, (2007).

[9] Y. Sun, S. Du, O. Gurewitz and D. Johnson, DW-MAC: a low latency, energy efficient demand wakeup MAC protocol for wireless sensor networks, Proceedings of the Ninth ACM International Symposium on Mobile Ad Hoc Networking and Computing (MobiHoc'08), pp. 53-62, May, (2008).

[10] K. Nguyen, Y. Ji and S. Yamada, Low Overhead MAC Protocol for Low Data Rate Wireless Sensor Networks, International Journal of Distributed Sensor Networks, vol. 2013, pp 9, (2013).

[11] Wireless LAN Medium Access Control (MAC) and Physical Layer (PHY) Specifications, IEEE Standard 802.11, (1999).

[12] S. C. Kim, J. H. Jeon and J. J. Kim, A Traffic-Adaptive Duty-Cycle MAC Protocol for Multi-hop Wireless Sensor Networks, NGC2014, (2014).

\section{Authors}

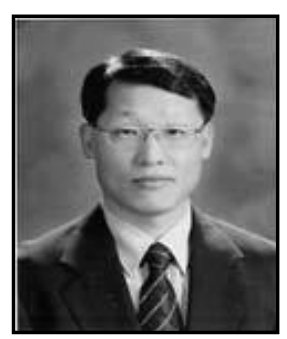

\section{Seong Cheol Kim}

1995. Polytechnic Institute of NYU Ph.D.

1997. 2. Sangmyung University, Dept. of Computer Science, professor

Research Interests: WSNs, Wireless LAN, Ubiquitous Computing

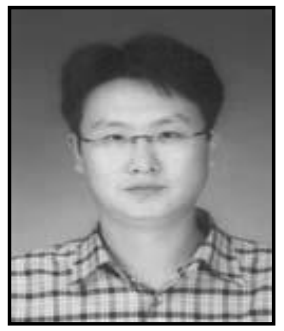

Jun Heon Jeon

2012.3. Sangmyung University, Dept. of Computer Science, Ph.D.

Research Interests: WSNs, MAC protocol, QoS, IoT 
International Journal of Multimedia and Ubiquitous Engineering Vol. 10, No. 2 (2015)

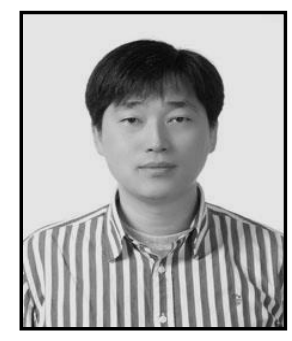

\section{Joong Jae Kim}

2013.3. Sangmyung University, Dept. of Computer Science, Ph.D.

Research Interests: WSNs, embedded software 